INTERNATIONAL JOURNAL OF SCIENTIFIC RESEARCH

\title{
AN ASSESSMENT OF AWARENESS, ATTITUDE, AND PRACTICE REGARDING \\ RELATIONSHIP BETWEEN DIABETES MELLITUS \& PERIODONTITIS AMONG GENERAL DENTAL \& MEDICAL PRACTITIONERS IN 3 MAJOR CITIES OF MADHYA PRADESH, INDIA- A SURVEY.
}

\section{Periodontology \\ Dr. Shrivastava Ratika \\ Dr. Jeevanand Deshmukh*}

\section{Dr. Khatri Richa}

Assistant professor, Department of Periodontology, Rishiraj College of Dental Sciences \& Research Centre, Bhopal, Madhya Pradesh, Pin number: 462-036

Professor \& Head, Department of Periodontology, Navodaya Dental College \& Hospital Raichur, Karnataka, Pin number: 584-103.*Corresponding Author

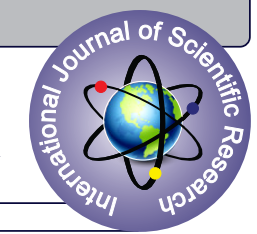

Ludhiana, Pin number: 14100

Dr. Asif K

Professor, Department of Periodontology, Navodaya Dental College \& Hospital, Raichur, Karnataka, Pin number: 584-103

Dr. Revu Das S D

Assistant Professor, Department of Periodontology, Sri Sankara Dental College \& Hospital, Akathumuri, Kerala, Pin Number: 695-318

Dr. Venkata Sai

Meghana M

Periodontist, Care super speciality Dental Clinic, 16/04/89, Pogathota, Venkataramana hotel street, Nellore, Andhra Pradesh, Pin Number: 524-001

\section{ABSTRACT}

Background: Diabetes mellitus and periodontal diseases evidently have a bidirectional relationship. To apply this into daily practise, an awareness among dentists and physicians of the same is imperative. Thus, a KAP (knowledge, attitude and practice) survey was conducted among dental and medical practitioners about the association between diabetes mellitus and periodontitis.

Methodology: A survey was conducted among 563 dental \& medical practitioners ( 263 physicians and 300 dentists) in 3 cities of Madhya Pradesh. Information on knowledge about complications related to periodontal conditions, attitude regarding diabetes and periodontal disease and the relation between the two was collected. Association between categorical was computed using Chi square test, with the $\alpha$-level set at 0.05 .

Results: Dentists were more aware about impact of diabetes complications on periodontal health as compared to physicians $(\mathrm{p}<0.05)$. Majority of dentists referred their patients to physicians for medical evaluation and were more interested in learning about bidirectional relationship between diabetes mellitus \& periodontal health, while fewer physicians referred their patients to dentists. The preferred mode of learning for dental practitioners was through conferences or CDEs, while medical practitioners preferred professional journals.

Conclusion: The knowledge, awareness and practice regarding bidirectional association between diabetes and periodontitis of dentists fared better than the physicians.

\section{KEYWORDS}

\section{Dental Practitioner, Diabetes Mellitus, Medical Practitioner, Periodontitis}

\section{INTRODUCTION}

Diabetes mellitus is 'chronic metabolic diseases characterized by hyperglycaemia resulting from defects in insulin secretion, insulin action, or both' as defined by the Expert committee of American Diabetes Association in 2003.[1]

The prevalence rate of diabetes mellitus globally is $55 \%$. More commonly seen in India, the World Health Organisation (WHO) reports show that 32 million people were suffering from this condition in the year 2000.[2] This number may gradually increase to 69.9 million by 2025 , as estimated by The International Diabetes Federation.[3] "India can thus be the diabetes capital of the world".[4]

Oral diseases, both gingival and periodontal, affect about $90 \%$ of the Indian population.[4] With the existence of a two- way relationship between diabetes mellitus and periodontitis, periodontitis has been labelled a common complications of diabetes.[5,6] Common pathophysiologic pathways involved in both diseases include low grade inflammation, altered host response, altered tissue homeostasis, and insulin resistance.[7]

Numerous studies support the view that the need for insulin in Insulin Dependent Diabetes Mellitus can be reduced through the systematic control of periodontal infection, either using mechanical therapy, or the administration of systemic antibiotics, hence improving the glycaemic control. $[8,9,10]$ Therefore, preventive measures and regular dental visits are of importance, hence avoiding complications as well as morbidity which is often associated with diabetes.[11]

A positive attitude and sound knowledge both physicians as well as dentists, are important to ensure overall good health of the patient. With a rapid rise in the number of diabetes cases, it is expected that health care providers will encounter greater number of patients with diabetes, and, in turn, periodontal complications. Till date, as per our knowledge, there is no literature to suggest knowledge of physicians and dentists in Madhya Pradesh as to the link between periodontitis and diabetes, even though a large number cases of diabetes are detected in Madhya Pradesh, especially in urban centres.[12] This study, therefore aims to assess and make aware both dental and medical practitioners in three major urban centres i.e. Bhopal, Indore and Jabalpur of Madhya Pradesh, highlighting the referral between the two specialities, hence contributing to the overall good health of an individual.

\section{METHODOLOGY}

A survey was conducted to assess the knowledge, attitude and practice regarding relationship between Diabetes Mellitus and Periodontitis among dentists and physicians in three major cities of Madhya Pradesh, namely Bhopal, Indore, and Jabalpur.

The institutional ethical committee, social justice and welfare department approval was obtained before commencement of study. At random, a combined total sample size of 600 Dental \& Medical Practitioners ( 300 dental \& 300 medical) were chosen, out of which 200 subjects (100 Dentists \& 100 Physicians) were selected from each city amounting to approximately $10-15 \%$ of registered practitioners. Data were collected from different types of settings including Private Medical and Dental Clinics, District Hospitals Dental \& Medical colleges as well as Primary Health Centers in 2013-2014 over a period of 3 months. Dental and medical practitioners were selected at random and requested to complete a self- structured questionnaire. Prior to inclusion, an informed verbal consent was obtained from all the participants. Dental and medical practitioners registered with Medical Council of India \& Dental Council of India and associated with academics as well as working in dental and medical colleges were included. Practitioners from other medical fraternity such as Homeopathy, Naturopathy, Ayurvedic etc were not included in the study. A questionnaire was formulated for recording the sociodemographic characteristics, knowledge of the bidirectional relation of diabetes and periodontal health, application of above knowledge in 
routine practice, interest and mode of learning about the link between the two diseases.

Participants were required to answer with choice of "Yes" or "No". Participants provided with the questionnaire were asked to return the questionnaire at the end of working hours or the next day.

An electronic database was used to store responses and analysis was done using a statistical software. Chi square test of association was used for categorical data, with the $\alpha$ level set at 0.05 .

\section{RESULTS}

A total of 563 dental \& medical practitioners (300 dentists \& 263 physicians) completed the questionnaire.

95.3\% dentists and $72.2 \%$ physicians were aware of the fact that periodontal abscess, gingival bleeding \& tooth mobility are frequently associated with diabetic patients. $82.3 \%$ dentists \& $48.6 \%$ physicians were aware of the fact that tooth loss is often associated with diabetic patients. The difference found between two categories was statistically significant $(\mathrm{P}<0.05)($ Graph 1$)$

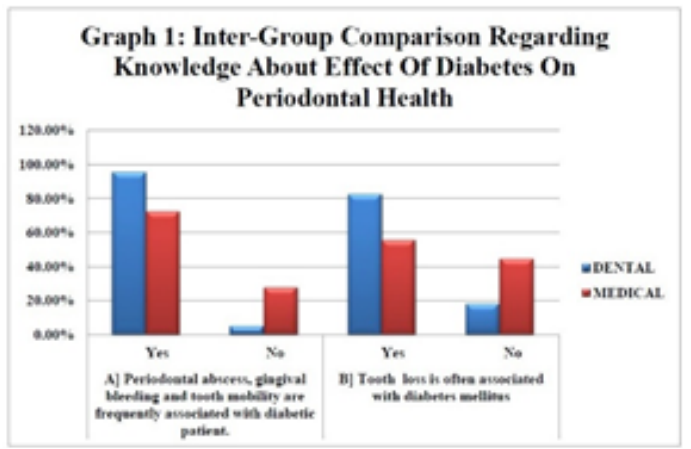

Graph 2 represents inter-group comparison regarding Knowledge \& Awareness about Bidirectional relation of Diabetes and Periodontal disease. The majority of the study participants were aware about the bidirectional relation of Diabetes Mellitus and Periodontal Disease and both make each other worse. $97 \%$ of dental practitioners \& $65 \%$ of medical practitioners said they will check for oral manifestations in diabetic patients in their routine practice with statistically significant difference $(\mathrm{P}<0.05)$.

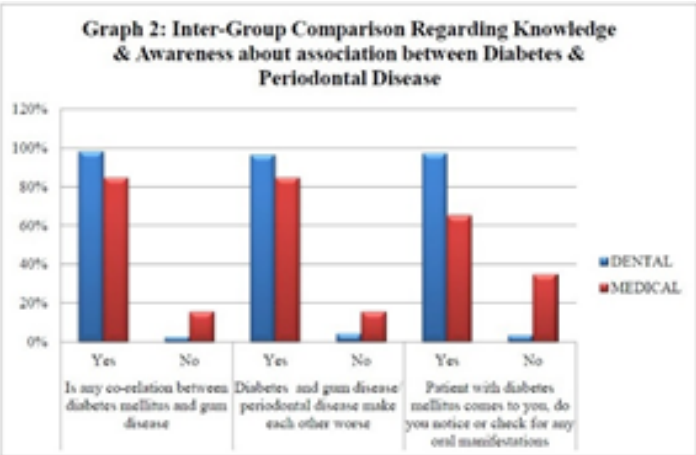

89.6\% Dental Practitioners referred their periodontitis patient for general blood glucose check-ups \& 79\% Medical Practitioners referred their poorly controlled diabetes patients to dentists $(\mathrm{P}<0.05)$. The awareness of how important frequent dental maintenance and scaling for patients whose diabetes is poorly controlled, was higher significantly in dentists than in physicians $(\mathrm{P}<0.05)(\mathrm{Graph} 3)$.

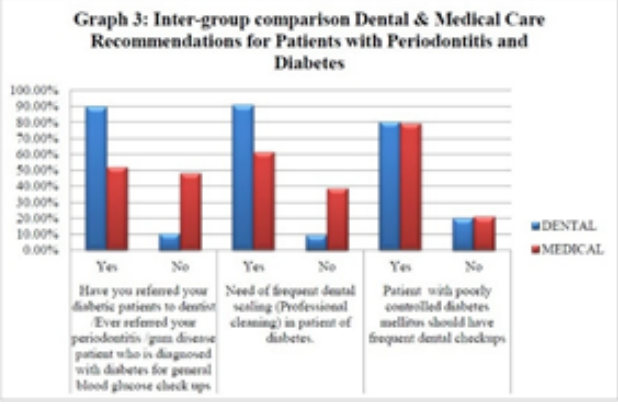

$67 \%$ dental practitioners and $29.6 \%$ medical practitioners attended continuing dental/medical education program regarding relation between periodontitis \& diabetes mellitus. $97.1 \%$ dental practitioners and $83.6 \%$ medical practitioners thought that the component of oral health should be merged with the diabetes component and incorporated as part of continuing education programme and $94 \%$ dental practitioners \& $86.3 \%$ medical practitioners were interested in learning more about diabetes mellitus \& its impact on oral health (Graph 4).

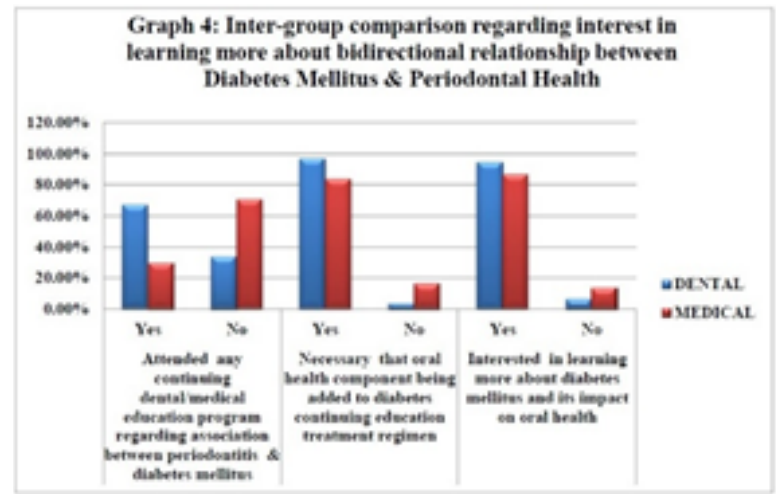

Medical Practitioners were interested in learning more about diabetes mellitus \& its impact on oral health through professional journals in comparison to those of Dental Practitioners interest in learning through conferences/CDEs (Graph 5).

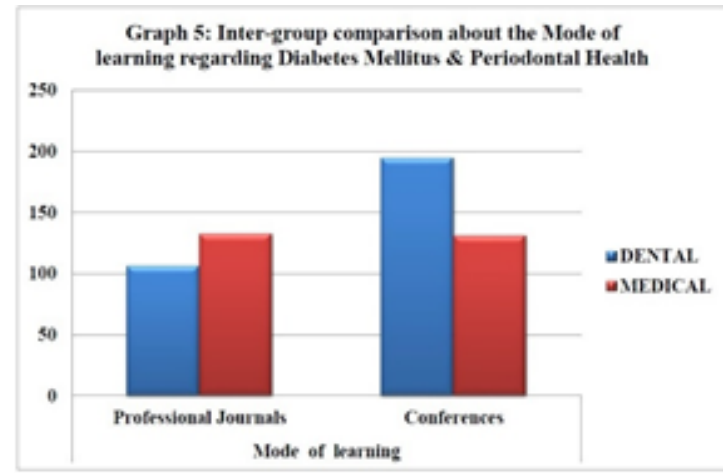

\section{DISCUSSION}

The association between diabetes mellitus and periodontal disease, with one condition leading to exacerbation of the other, is not unknown.[13] There is a need to increase the knowledge of this link among health professionals.[14] This is turn may result in an improved attitude and behaviour towards the management of their respective patients.[15]

It has been proved that periodontal complications may have a higher risk of developing in diabetics, which include conditions such as gingival inflammation and bleeding, tooth mobility, periodontal abscess, and loss of teeth.[16] Overall $84.5 \%$ of practitioners in our study were aware about the fact that periodontal abscess, gingival bleeding and tooth mobility are frequently seen in diabetic patients. However, as compared to physicians, awareness of dentists was higher. This is similar to a cross sectional survey report, where in, when compared to physicians, dentists were more aware about the periodontal complications in diabetic patients.[17]

Periodontal diseases and followed by the loss of teeth hamper the quality of life, while affecting general health of individuals. A larger number of dentists agreed that tooth loss due to periodontitis is associated with diabetes. As per a cross sectional survey, overall $50 \%$ respondents and $49.1 \%$ of physicians were in agreement that tooth loss is frequently seen in diabetic individuals. It is clear from the abovementioned studies that there is a paucity in knowledge regarding the effects diabetes may have on periodontal health among physicians globally, however physicians in our study fared slightly better than the others.[17]

Unless the medical fraternity is knowledgeable of this bidirectional relationship, it is not possible for them to counsel their patients accordingly.[18] 
Diabetic patients are at a high risk to develop periodontitis.[19] In turn, poor glycaemic control may result from severe periodontitis,[20] hence treating them may help control sugar levels.[21,22] In our study, most of the study subjects were aware about the bidirectional relation between the two conditions, and agreed that their combined presence makes each other worse. However, on intergroup comparison, dentists were more aware than the physicians. Only $65 \%$ of physicians said they check for oral manifestations in diabetic patients compared to $97 \%$ of dentists.

Most of the subjects are never told by their endocrinologist to get regular dental check-ups. Studies show physicians are unable to inform their patients regarding the implications of diabetes on oral health, as they lack sufficient knowledge on this topic themselves.[23]

Incoming medical interns have shown inadequate knowledge regarding bidirectional relationship.[24,25,26] A study conducted in Chennai showed good knowledge of dentistry among interns and medical practitioners. This may be due to dental health being included as a part of their medical graduation syllabus. This also applies to different medical colleges in different Indian States as the same syllabus is followed as suggested by Medical Council of India (MCI). Probably because of the inclusion of dental syllabus as a part in medical curriculum, a significantly better response from physicians in our study was obtained as compared to other studies.[27] Formal training is a significant factor that affects the attitudes of health care providers towards managing diabetics and the provision of advice to such patients about periodontal risk associated with diabetes.[28]

The effects of poor oral health on general health was studied by A Gur et al (2008), to evaluate their knowledge on the impact of oral health on general health. He referral rate of patients with systemic disorders associated with dental diseases to dentists was only $12 \%$.[29] Robertson et al (2003) stressed that it is important to educate and inform physicians the importance of referring patients with diabetes to a dentist.[20] Hein (2006) opined that the human body should be treated as a whole, without considering the oral health and general health components separately, for which an interdisciplinary approach is necessary.[30] Sreenivas Nagarkanti (2013) suggested that if medical practitioners refer their patients, the access to oral health care will improve.[31]

Many physicians concentrate only on systemic-health conditions, and feel that they are not responsible for managing oral health care. In a survey, physicians have reported lack of information on oral health problems related to diabetes and their involvement in oral health implication for diabetic patients.[17] Therefore, enhancing communication between the two branches of medicine and dentistry providers is essential to facilitate an exchange of knowledge between the two professions to provide an effective management of serious chronic diseases such as DM.

From our study, it was noted that between dentists and physicians, dentists are more interested in learning about the link between diabetes mellitus \& periodontal health. However, number of dentists and physicians attending continuing dental/medical education programmes was on lower side. It is essential to evaluate the dental/medical professional knowledge which in turn will assist in providing appropriate level of Continuing Dental Education Programme.

In a study done by Mary H Lopes (2012) regarding certified diabetes educator knowledge on the relationship between diabetes and periodontitis, an overwhelming number of people said that oral bacteria thrives in a high sugar environment, and is worsened in the presence of periodontal disease, suggesting better knowledge among certified diabetes educators about the bidirectional relationship. This they attributed to the inclusion of oral health in their curriculum and also as part of their continuing education programmes. [32] Yuen et al (2010) reported that when the curriculum includes an oral health component, it helps reinforce CDEs recommendations for frequent scaling, and their counselling about the impact their uncontrolled diabetes can have on the periodontium.[33] Sreenivas Nagarakanti (2013) suggested that medical colleges and continuing medical education programs should emphasize the role of physicians as contributors towards oral health.[31] There is clearly a scope, to develop a better support system that promotes coordination among the different specialities such as dental and medical, involved in the care of diabetic individuals.[34]
$35.6 \%$ of dental practitioners and $50.1 \%$ of medical practitioners were interested in learning more about diabetes mellitus \& its impact on oral health through professional journals in comparison of $64.6 \%$ of dental practitioners \& $49.8 \%$ of medical practitioners, interested in learning through conferences. Majority of medical practitioners were interested in learning through professional journal probably because of their busy schedule and round the clock requirement of their services towards their patients as attending conferences entails more time period.

Mary Lopes (2012) conducted KAP study in the university of North Carolina among certified diabetes educators (CDEs) who said that $80 \%$ knowledge about oral health is obtained from speciality journals.[35] This finding is similar to the study by Koerber et al (2006) who found that workplace guidelines and protocols and speciality journals are ideal to educate nurses and nutritionists and update them about periodontal diseases.[36]

Children and adults frequent physicians more than dentists, hence they have the advantage to reach out to a larger population and provide dental counselling. [31] The dentist Knowledge, Attitude and Practice (KAP) in our study about Periodontal Disease and Diabetes Mellitus was better in all aspects as compared to physicians and its responsibility of dentists to sensitize medical practitioners towards periodontal awareness. However, medical practitioners included in our study fared better in all aspects when compared to other similar studies reported in the literature.

\section{CONCLUSION}

Our study relied on self-reported data, which may be a limitation, yet we have taken several steps to ensure their correctness as described in material methods. Sampling frames included both registered dental and medical practitioners. Maximum response was ensured by regular follow- up, hence $100 \%$ response rate for dentists and $87.6 \%$ response rate of physicians was ensured

\section{REFERENCES}

Hogan P, Dall T, Nikolov P. Economic costs of diabetes in the U.S. in 2002. Diabetes Care 2003;26:917-32

Wild S, Roglic G, Green A, Sicree R, King H. Global prevalence of diabetes: estimates for the year 2000 and projections for 2030. Diabetes Care 2004;27:1047-53.

Joshi SR, Parikh RM. India--diabetes capital of the world: now heading towards hypertension. JAssoc Physicians India 2007;55:323-4

Shaju JP, Zade RM, Das M. Prevalence of periodontitis in the Indian population: A literature review. J Indian Soc Periodontol 2011;15:29-34

5. Loe H. Periodontal disease. The sixth complication of diabetes mellitus. Diabetes Care $1993 ; 16: 329-34$

6. Taylor GW, Burt BA, Becker MP, Genco RJ, Shlossman M, Knowler WC, et al. Severe periodontitis and risk for poor glycemic control in patients with non-insulin-dependen diabetes mellitus. J Periodontol 1996;67:1085-93.

7. Miller LS, Manwell MA, Newbold D, Reding ME, Rasheed A, Blodgett J, et al. The relationship between reduction in periodontal inflammation and diabetic control: a repost of 9 cases. J Periodontol 1992;63:843-8.

8. Darre L, Verges JN, Gourdy P, Sixbon M. Efficacy of periodontal treatment on glycemic control in diabetic patient: A meta-analysis of interventional studies. Diabetes Metab 2008:34:497-506

9. Madden TE, Herriges B, Boyd L, Laughlin G, Chlodo G, Rosentein DI. Alterations in HbA1C following minimal or enhanced non-surgical, non antibiotic treatment of gingivitis or mild periodontitis in type 2 diabetic patients: A Pilot trial. J Contemp Den Pract 2008;9:9-16.

10. Oliver RC, Tervonen T. Diabetes--a risk factor for periodontitis in adults. J Periodontol $1994: 65 \cdot 530-8$

11. Yasmeen BR, Ullah KA, Dil R, Ahamed MM. Diabetic patients; level of awareness about oral health knowledge, attitude and practices. Pakistan Oral \& Dental Journal 2011;31:292-5.

12. Ramachandran A, Snehalatha C, Dharmaraj D, Viswanathan M. Prevalence of glucose tolerance in Asian Indians. Urban-rural difference and significance of upper body adiposity. Diabetes Care 1992;15:1348-55

13. Mealey BL, Oates TW. Diabetes mellitus and periodontal diseases. J Periodontol 2006; 77:1289-303.

14. Kunzel C, Lalla E, Lamster I. Dentist's management of the diabetic patient: Contrasting generalists and specialists. Am J Public Health 2007;97:725-30.

15. Taiyeb-AliTB, Raman RP, Vaithilingam RD Relationship between periodontal disease and diabetes mellitus: An Asian perspective. Periodontol 2000 2011:56:258-68.

16. Al-Khabbaz AK, Al-Shammari KF. Diabetes mellitus and periodontal health: dentists knowledge. Med Princ Pract 2011;20:538-44.

17. Al-Khabbaz AK, Al-Shammari KF, Al-Saleh NA. Knowledge about the association between periodontal diseases and diabetes mellitus: contrasting dentists and physicians. JPeriodontal 2011;82:360-6.

18. Allen EM, Ziada HM, O'Halloran D, Clerehugh V, Allen PF. Attitudes, awareness an oral health-related quality of life in patients with diabetes. J Oral Rehabil 2008;35:218-23.

19. Johnson NW, Griffiths GS, Wilton JM, Maiden MF, Curtis MA, Gillett IR, et al. Detection of high-risk groups and individuals for periodontal diseases. Evidence for the Detection of high-risk groups and individuals for periodontal diseases. Evidence for the Pexionee of high-risk groups a

20. Robertson C, Drexler AJ, Vernillo AT. Update on diabetes diagnosis and management. J Am Dent Assoc 2003;134:16-23.

21. Kiran M, Arpak N, Unsal E, Erdogan MF. The effect of improved periodontal health on metabolic control in type 2 diabetes mellitus. J Clin Periodontol 2005;32:266-72.

22. Jones JA, Miller DR, Wehler CJ, Rich SE, Krall-Kaye EA, McCoy LC, et al. Does periodontal care improve glycemic control? The department of Veterans affairs dental diabetes study. J Clin Periodontol 2007;34:46-52. 
23. Smith RM, Fleming LE, Christopher L. Arheart, KL, Wilkinson JD. Periodontal disease and diabetes: Knowledge and attitudes assessment project. Florida Public Health Review $2007 ; 4: 12-7$

24. Asa'ad F, Al-Maflehi N, Alelyan B, Assad L, Alrumaih W, Alassad F, et al. Knowledge and orientations of medical interns toward periodontal disease in Saudi Arabia. Saudi J Oral Sci 2014;1:98-104

25. Quijano A, Shah AJ, Schwarcz AI, Lalla E, Ostfeld RJ. Knowledge and orientations of internal medicine trainees toward periodontal disease. J Periodontol 2010;81:359-63.

26. Bissett SM, Stone KM, Rapley T, Preshaw PM. An exploratory qualitative interview study about collaboration between medicine and dentistry in relation to diabetes management. BMJ open 2013;3:1-7.

27. Srinidhi S, Ingle NA, Chaly PE, Reddy C. Dental awareness and attitudes among medical practitioners in Chennai. J Oral Health Comm Dent 2011;5:73-8.

28. Petersen PE. Global policy for improvement of oral health in the 21 st centuryimplications to oral health research of World Health Assembly 2007, World Health Organization. Community Dent Oral Epidemiol 2009;37:1-8

29. Gur A, Majra JP. Knowledge, attitude, practices regarding the systemic effects of oral diseases among the medical practitioners. Int J Dent Sciences 2008;6:1-5.

30. Hein C, Small D. Combating diabetes, obesity, periodontal disease and interrelated inflammatory conditions with a syndemic approach. Grand Rounds Oral-Sys Med 2006;2:36-47

31. Nagarakanti S, Epari V, Athuluru D. Knowledge, attitude, and practice of medical doctors towards periodontal disease. J Indian Soc Periodontol 2013;17:137-9.

32. Corbet EF, Leung WK. Epidemiology of periodontitis in the Asia and Oceanic regions. Periodontol 2000 2011;56:25-64.

33. Albandar JM, Rams TE. Global epidemiology of periodontal diseases: an overview. Periodontology 2000 2002;29:7-10.

34. Malik G, Lehl G, Talwar M. Association of Periodontitis with Diabetes Mellitus: A review. J Medical College Chandigarh 2011;1:10-4.

35. Lopes MH, Southerland JH, Buse JB, Malone RM, Wilder RS. Diabetes educators' knowledge, Opinions and behaviors regarding periodontal disease and diabetes. J Dent Hyg 2012;86:82-90.

36. Yuen HK, Onicescu G, Hill EG, Jenkins C. A survey of oral health education provided by certified diabetes educators. Diabetes Res Clin Pract 2010;88:48-55. 